\title{
Gas Chromatography-Mass Spectrometry (GC-MS/MS) Analysis, Ultrasonic Assisted Extraction, Antibacterial and Antifungal Activity of Emblica officinalis Fruit Extract
}

\author{
Ali Mohammed Mohammed Ali Al-Samman', Kahkashan², Nadeem Ahmad Siddique ${ }^{1, *}$
}

\section{Ali Mohammed Moham- med Ali Al-Samman', Kahkashan², Nadeem Ah- mad Siddique ${ }^{1, *}$}

\section{'Bioactive Natural Compound Laboratory (BNCL); Department of Pharmacognosy and Phytochemistry, Glocal School of Pharmacy, Glocal University, Saharanpur-247121, Uttar Pradesh, INDIA. \\ ${ }^{2}$ Department of Computer Science and Bio Informatics, Faculty of Natural Science. Jamia Millia Islamia, \\ New Delhi, Delhi- 110025, INDIA. \\ Correspondence \\ Dr. Nadeem Ahmad Siddique}

Bioactive Natural Compound Laboratory (BNCL); Department of Pharmacognosy and Phytochemistry, Glocal School of

Pharmacy, Glocal University, Saharanpur247121, Uttar Pradesh, INDIA.

Phone no : +91 8954006731

E-mail: siddique.nadeem567@gmail.com History

- Submission Date: 24-10-2018;

- Review completed: 27-11-2018;

- Accepted Date: 30-11-2018.

DOI : 10.5530/pj.2019.11.47

Article Available online

http://www.phcogj.com/v11/i2

\section{Copyright}

(C) 2019 Phcog.Net. This is an openaccess article distributed under the terms of the Creative Commons Attribution 4.0 International license.

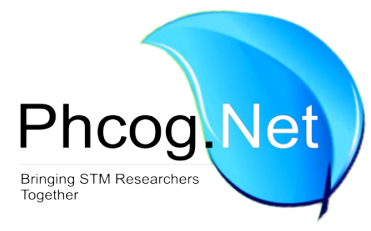

\begin{abstract}
Introduction: Emblica officinalis is one of the common plants being used traditionally in different ways to search for cures and relief from various diseases. Among these diseases, Emblica officinalis had many of medicinal uses in treating a wide variety of bacterial and fungal infections. In this study, Ultrasonic assisted extraction (UAE) was efficiently used for the preparation of aqueous and methanolic extracts of Embelica officinalis fruits (EOFE). The phytoconstituents was detected through GC-MS/MS analysis to confirm antimicrobial effect of EOFE. Materials and Methods: The extraction was carried out at $20^{\circ} \mathrm{C}$, for 20 min using a solid-to-solvent ratio of 1: $60 \mathrm{w} / \mathrm{v}$. The extracts were subjected to GC-MS/MS analysis. The in-vitro effect of extracts against twenty one microbial strains was investigated by an agar well diffusion method in different concentrations $(25 \mu \mathrm{g} / \mathrm{mL}-1000 \mu \mathrm{g} / \mathrm{mL})$. Results: The percentage yield was found to be $41.33 \% \mathrm{w} / \mathrm{v}$ and $23.0 \% \mathrm{w} / \mathrm{v}$ with water and methanol, respectively. Phenol,3,5-bis1,1 dimethylethyl, 2,4-di-tert-butylphenol and heptasiloxa ne1,1,3,3,5,5,7,7,9,9,11,11,13,13-tetradecamethyl were confirmed by GC-MS/MS analysis. Effectiveness of extracts against Gram negative bacteria; Providencia alcalifaciens and Gram positive bacteria; Bacillus pumilis, Bacillus polymyxa and fungal strains; Neurospora crassa, Aspergillus brasileinsis and Cladosporium oxysporum are reported for the first time. Aqueous extract revealed excellent antibacterial activity at $50 \mu \mathrm{g} / \mathrm{mL}$ and antifungal activity at $100 \mu \mathrm{g} / \mathrm{mL}$ whereas methanolic extract showed antifungal activity at $250 \mu \mathrm{g} / \mathrm{mL}$. Conclusion: Results highlight the considerable inhibitory effect of EOFE against various microbial species was mainly due to the presence of phenolic compounds and other phytocompounds.
\end{abstract}

Key words: GC-MS/MS, Emblica officinalis, Ultrasonic Assisted Extraction, Antibacterial, Antifungal.

\section{INTRODUCTION}

The plant Emblica officinalis has the potential for wide therapeutic applications (Family Euphorbiaceae), commonly known as amla. It is reported E. officinalis fruit is widely producing bioactive molecules which are found to be useful for the treatment of various diseases including antibacterial, antifungal, antiviral, immunomodulatory, analgesic, antipyretic, lowering cholesterol level, etc. ${ }^{1}$ E. officinalis seems to be a rich source of vitamin- $\mathrm{C}$, amino acids and minerals like chromium, zinc and copper. Phenolic compounds generally distributed in E. officinalis, so it is consider to be a promising source of hydrolysable tannins, Emblicanin A and B, gallic acid and ellagic acid. ${ }^{2}$ According to World Health Organization (WHO), today most of the drugs are obtained from medicinal plants. Bacteria and fungi are widely scattered as environmental contaminants and nearly $10 \%$ of people worldwide have fungal allergy. ${ }^{3}$ The secondary metabolism of the plant such as E. officinalis and Coriandrum sativum, ${ }^{4}$ Aloe vera and Vitex negundo, ${ }^{5}$ produce compounds which give plant antibacterial and antifungal character. Various methods are claimed to carry out the extraction of active constituents from plant material however several factors are considered for extraction such as effectiveness, operating cost, simplicity of method and waste production. Many conventional extraction techniques such as Soxhlet, heated reflux and cold pressing, were used for the extraction from medicinal plants. However, ultrasonic assisted extraction (UAE) is a novel technique used to extract bioactive compounds from medicinal plants. This is an efficient method that, requires a very short time, less solvent, reduces the discharge of hazardous pollutants through reducing organic and synthetic solvent overexposure, eco-friendly and is comparatively simple to operate. It reduces processing time, minimal risk of oxidation, disintegration and decomposition of chemical constituents of plant. ${ }^{6}$ Because of these excellent advantages, the UAE techniques have become one of the best techniques
Cite this article: Al-Samman AMMA, Kahkashan, Siddique NA. Gas Chromatography-Mass Spectrometry (GC-MS/MS) Analysis, Ultrasonic Assisted Extraction, Antibacterial and Antifungal Activity of Emblica officinalis Fruit Extract. Pharmacog J. 2019;11(2):315-23. 
for the extraction of bioactive constituents from medicinal plants. ${ }^{7-8} \mathrm{UAE}$ may be explored to extract the vital bioactive constituents of E. officinalis. GC-MS/MS is an analytical technique used for identifying the bioactive compounds from plant extract. Pyrogallol, 5-hydroxymethylfurfural, 5-methyl-2-furylmethyl ketone, Gallic-acid as major constituents in leaves of E. officinalis identified by Gas chromatography-mass spectrometry (GC-MS). ${ }^{9}$ The aim of this current study is to analyse the components of E. officinalis fruit by Ultrasonic Assisted Extraction (UAE) in different solvents by using GC-MS/MS and investigate the in-vitro effects of it against bacterial and fungal strain. Although antimicrobial activity of E. officinalis fruit extract reported to date, for the best of our knowledge there are still bacterial and fungal strains for which antimicrobial activity are leaked in the previous studies. Hence our study increasingly turning its contribution to folk medicines, looking for new leads to develop better drugs against microbial infections. Further, UAE explore to extract the vital bioactive constituents whilst structure of HSTDM was elucidated first time in EOFE by GC-MS/MS analysis.

\section{MATERIALS AND METHODS}

\section{Equipment and chemicals}

The Extraction was performed on an ultrasonic bath (GT Sonic-1990Q Ts, China, Ultrasonic Power $200 \mathrm{~W}$ and Frequency $33 / 40 \mathrm{kHz}$ ). The Analysis was performed on Agilent 7000D GC-MS/MS (Triple Quad) system. Methanol was supplied by Merck (Darmstadt, Germany). All other inorganic chemicals and organic solvents were of reagent grade.

\section{Plant material}

Fresh sample of E. officinalis were collected from Panchsheel Park-South Delhi, 110017, India, Plant was authenticated by National Herbarium of Cultivated Plants (NHCP), Pusa campus, New Delhi-110012 and specimen (NHCP/NBPGR/2017-1) was submitted to Bioactive Natural Compound Laboratory (BNCL), Department of Pharmacognosy and Phytochemistry, Glocal school of Pharmacy, Glocal University. The plant material initially washed thoroughly to remove impurities and was chopped in to small pieces with a blender and then placed in to shade to dry at room temperature. The shade dried plant materials were finely grained into powder using an electric grinder and store in airtight bottles for further investigations.

\section{Preparation of extracts Ultrasonic Assisted Extraction (UAE)}

The dried plant material (10 gm) was submitted to extraction with water and methanol $(60 \mathrm{~mL})$ using UAE apparatus (GT Sonic- 1990Q Ts, China) equipped with ultrasonic bath, digital meter and a temperature regulator. The device was operated at a frequency of $33 / 40 \mathrm{kHz}$, an ultrasonic input power of $200 \mathrm{~W}$ and the procedure was repeated for three times. Ultrasonication generates massive heat; to avoid this bath was run at room temperature $\left(25^{\circ} \mathrm{C}\right)$. The extract was filtered and concentrated using rotary evaporator (Buchi, Switzerland) to remove the solvent completely.

\section{Chromatographic Analysis \\ Identification of component in EOFE using Gas chromatography-mass spectrometry (GC-MS/MS) Preparation of samples}

EOFE was submitted to GC-MS/MS evaluation and the sample was prepared by taken $10 \mathrm{mg}$ of extract in volumetric flask and adjusted the volume up to $10 \mathrm{~mL}$ with water and methanol followed by centrifugation at $5000 \mathrm{rpm}$ for $5 \mathrm{~min}$. Supernatant was filtered through $0.22 \mu \mathrm{m}$ syringe and $1 \mathrm{~mL}$ of this solution was taken for GCMS/MS analysis.

\section{GC-MS/MS conditions}

The constituents in aqueous and methanolic extract of EOFE were examined on Agilent 7000D GC-MS/MS (Triple Quad - S. No. US1710UM1) system equipped with HP-5 MS capillary column with a $5 \%$ phenyl polysiloxane stationary phase $(30.0 \mathrm{~m} \times 0.25 \mathrm{~mm}$, film thickness $0.25 \mu \mathrm{m})$. Different temperatures of oven were considered to achieve good separation in standard as well as in samples. The instrument was set to an initial temperature of $70^{\circ} \mathrm{C}$ and (maintained for $2 \mathrm{~min}$ ) then in second stage temperature was allowed to raise by $3^{\circ} \mathrm{C} / \mathrm{min}$ up to $150^{\circ} \mathrm{C}$, then finally attended to $200^{\circ} \mathrm{C}$ by expanding the heating rate of $8^{\circ} \mathrm{C} / \mathrm{min}$. The flow rate of Helium carrier gas was kept $1.0 \mathrm{~mL} / \mathrm{min}$, injection port temperature was kept at $280^{\circ} \mathrm{C}$ and pressure was maintained 60 Kilo Pascal while the ionization voltage was $70 \mathrm{eV}$. Mass spectra were examined in scanning mode at $25-500 \mathrm{~m} / \mathrm{z}$. Interpretation of GC-MS/MS spectra were, moreover, recognized and verified by using the NIST 14-MS database library stored in Standard Analytical Laboratory (SAL). The name, Molecular Weight (MW), Retention Time (RT), peak area and structure of the active constituents of EOFE were ascertained.

\section{Selection of microorganisms Bacterial strains}

Seven Gram negative bacteria Pseudomonas aeruginosa (MTCC 7837), Escherichia coli (MTCC 723), Vibrio cholera (MTCC 3906), Salmonella typhi (MTCC 3216), Shigella dysenteriae (MTCC 1842), Proteus mirabilis (MTCC 743), Providencia alcalifaciens (MTCC 4430), seven Gram positive Staphylococcus aureus (MTCC 3160), Bacillus subtilis (MTCC 736), Bacillus megaterium (MTCC 4911), Bacillus pumilis (MTCC 1607), Bacillus cereus (MTCC 430), Staphylococcus pyogenous (MTCC 1927) and Bacillus polymyxa (MTCC 122) were used in the present study. Subsequent specification of Gram negative bacteria; Providencia alcalifaciens and Gram positive bacteria; Bacillus pumilis and Bacillus polymyxa were reported first time in our study.

\section{Fungal strain}

Seven pathogenic fungi Aspergillus niger (MTCC 1881), Neurospora crassa (MTCC 1855), Penicillium chrysogenum (MTCC 6891), Trichoderma viridae (MTCC 4329), Aspergillus brasileinsis (MTCC 1344), Candida albicans (MTCC 227) and Cladosporium oxysporum (MTCC 1777) were used in the present study. In order to antifungal study three new fungal strains Neurospora crassa, Aspergillus brasileinsis and Cladosporium oxysporum were reported first time in our study.

\section{Antibacterial activity}

Antibacterial activity was evaluated by agar-well diffusion assay technique. Initially, the potential of stock cultures of bacteria were checked by inoculating in broth media (Peptone-10 gm, NaCl-10 gm and Yeast extract- $5 \mathrm{gm}$, Agar- $20 \mathrm{gm}$ in $1000 \mathrm{~mL}$ of distilled water) and grown at $37^{\circ} \mathrm{C}$ for $18 \mathrm{~h}$. The wells were prepared on Agar plates which was subsequently inoculated with $18 \mathrm{~h}$ old cultures $(100 \mu \mathrm{l}, 104 \mathrm{CFU})$ and swabbed uniformly. Latter on $20 \mathrm{~min}$, the wells were filled with extract at different concentration. Ciprofloxacin was inoculated as positive control with different concentrations $(25,50,100,250,500$ and $1000 \mu \mathrm{g} / \mathrm{mL})$ while DMSO (Dimethyl sulfoxide) $2 \%$ solution was used as negative control. After addition of media, the plates were incubated at $37^{\circ} \mathrm{C}$ for $24 \mathrm{~h}$ and MIC (concentration at which no optically clear growth showed) were determined the zone of inhibition. Tests for in-vitro antibacterial activity were performed in triplicate and calculated mean values.

\section{Antifungal activity}

Czapek-Dox agar media were used for evaluating antifungal activity. Initially, the stock cultures of fungi were cheched by inoculating in broth 
media; sucrose $(30.0 \mathrm{gm} / \mathrm{mL})$, Sodium nitrate $(2.0 \mathrm{gm} / \mathrm{mL}), \mathrm{K}_{2} \mathrm{HPO}_{4}$ $(1.0 \mathrm{gm} / \mathrm{mL}), \mathrm{MgSO}_{4} .7 \mathrm{H}_{2} \mathrm{O}(0.5 \mathrm{gm} / \mathrm{mL}), \mathrm{KCl}(0.5 \mathrm{gm} / \mathrm{mL}), \mathrm{FeSO}_{4}(0.01$ $\mathrm{gm} / \mathrm{mL})$ and Agar $(20 \mathrm{gm} / \mathrm{mL})$ and grown at $37^{\circ} \mathrm{C}$ for $48 \mathrm{~h}$. The wells were prepared on Agar plates which was subsequently inoculated with $48 \mathrm{~h}$ old cultures $(100 \mu \mathrm{l}, 104 \mathrm{CFU})$ and swabbed uniformly. Then after $20 \mathrm{~min}$, the wells were filled with different concentrations of samples. Amphotericin was inoculated as positive control with different concentrations $(25,50,100,250,500$ and $1000 \mu \mathrm{g} / \mathrm{mL})$ to compare the activities of EOFE at the same concentrations while DMSO 2\% solution was used as negative control. After addition of media, all plates were incubated at $37^{\circ} \mathrm{C}$ for $96 \mathrm{~h}$ and the diameter zones of inhibition were noted. Tests for in-vitro antifungal activity were performed in triplicate and calculated mean values.

\section{RESULTS}

\section{Selection of Ultrasonic Assisted Extraction (UAE)}

The conventional methods normally performed at high temperature for several hours and a large amount of solvents are the main disadvantage of conventional technique which was found not economically suitable for the extraction purpose,${ }^{10-11}$ in which food is subjected to the elevated pressure which is mostly between 100 to $800 \mathrm{MPa}$. HHPP is seen not only in food engineering, but also have other application areas, such as extraction of active ingredients from natural biomaterials. In this study, several extraction conditions such as two different solvents [methanol and solvent cocktail ( $\mathrm{dH}$ 2O:ethanol:methanol:acetone: $\mathrm{CH}_{2} \mathrm{CI}_{2}$ - 1:2.5:2.5:2:2 in this study using UAE method high percentage yield of EOFE was found in water $(41.33 \mathrm{w} / \mathrm{v})$ and methanol $(23.00 \mathrm{w} / \mathrm{v})$ at low temperature $\left(25^{\circ} \mathrm{C}\right)$. Moreover, a few minutes of extraction time $(20 \mathrm{~min})$ and small amount of extraction solvent $(60 \mathrm{~mL})$ were required.

\section{Optimization of chromatographic conditions for GC-MS/ MS}

Different temperature ramping were tried to achieve good separation of constituents in EOFE. The oven temperature was initially $70^{\circ} \mathrm{C}$ for $2 \mathrm{~min}$. The temperature was increased to $150^{\circ} \mathrm{C}$ at rate of $3^{\circ} \mathrm{C} / \mathrm{min}$ and then gradually increased to $200^{\circ} \mathrm{C}$ at a rate of $8^{\circ} \mathrm{C} / \mathrm{min}$. Good separation was found when inlet temperature $\left(70^{\circ} \mathrm{C}\right)$, helium flow rate $(1.0 \mathrm{~mL} / \mathrm{min})$, total running time $(41.87 \mathrm{~min})$ while the ionization voltage was $70 \mathrm{eV}$ and MS were operated in SCAN mode.

\section{Peak identification}

Molecular characterization and elucidation by GC-MS/MS spectra was conducted using the database of Standard Analytical Laboratory (SAL). The compounds in EOFE were recognized by their Retention Time (RT), Molecular Weight (MW), molecular structure and peak area in percentage which revealed good separation and structure of compounds.

\section{Determination of different compound by GC-MS/ MS}

GC-MS/MS chromatogram of methanolic and aqueous extract of E. officinalis were given a well separated peaks of PBDME at Rt (8.5418.614 $\mathrm{min})$, DTBP at Rt (8.541-8.607 $\mathrm{min})$ and HSTDM at Rt (36.199$36.00 \mathrm{~min}$ ), as shown in [Figures 1, 2 and 3] respectively. The column

Table 1: Description and relative composition of active constituents of $E$. officinalis fruit extract by GC- MS/MS.

\begin{tabular}{|c|c|c|c|c|c|}
\hline E. officinalis fruit extract & Name of compound & Formula & Mol. Wt. (gm/mol) & Peaks & RT (min) \\
\hline Methanolic extract & Phenol, 3,5-bis (1,1-dimethylethyl) & $\mathrm{C}_{14} \mathrm{H}_{22} \mathrm{O}$ & 206 & 24 & 8.5 \\
\hline \multirow[t]{2}{*}{ Aqueous extract } & 2,4-Di-tert-butylphenol & $\mathrm{C}_{14} \mathrm{H}_{22} \mathrm{O}$ & 206 & 15 & 8.6 \\
\hline & Heptasiloxane, $1,1,3,3,5,5,7,7,9,9,11,11,13,13$-tetradecamethyl & $\mathrm{C}_{14} \mathrm{H}_{44} \mathrm{O}_{6 \mathrm{Si}}$ & 503 & 29 & 36.2 \\
\hline
\end{tabular}

RT: Retention Time; Mol. Wt.: (Molecular weight)

Table 2: Antibacterial activity of methanolic fruit extract of $E$. officinalis on Gram negative and Gram positive bacteria.

\begin{tabular}{|c|c|c|c|c|c|c|c|c|}
\hline \multicolumn{2}{|c|}{ Types and name of organisms } & \multicolumn{7}{|c|}{ Concentration of extract $(\mu \mathrm{g} / \mathrm{mL})$ and Zone of inhibition $(\mathrm{mm})^{\mathrm{a}} \pm$ SD } \\
\hline & & $25 \mu \mathrm{g}$ & $50 \mu \mathrm{g}$ & $100 \mu \mathrm{g}$ & $250 \mu \mathrm{g}$ & $500 \mu \mathrm{g}$ & $1000 \mu \mathrm{g}$ & MIC $\mu \mathrm{g}$ \\
\hline \multirow{7}{*}{ 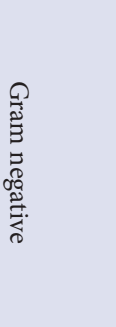 } & E. coli & NS & NS & NS & NS & $4.1 \pm 0.9$ & $15.9 \pm 0.8$ & 500 \\
\hline & P. aeruginosa & NS & NS & NS & $10.2 \pm 0.6$ & $15.2 \pm 0.6$ & $18.3 \pm 0.4$ & 250 \\
\hline & V. cholerae & NS & NS & NS & NS & $12.5 \pm 0.4$ & $13.2 \pm 0.7$ & 500 \\
\hline & S. typhi & NS & NS & NS & $10.5 \pm 0.8$ & $13.9 \pm 0.8$ & $16.3 \pm 0.7$ & 250 \\
\hline & S. dysenteriae & NS & NS & NS & NS & $5.7 \pm 0.7$ & $9.8 \pm 0.7$ & 500 \\
\hline & P. mirabilis & NS & $6.2 \pm 0.8$ & $8.7 \pm 0.6$ & $11.1 \pm 0.8$ & $12.4 \pm 0.4$ & $12.9 \pm 0.8$ & 50 \\
\hline & P. alcalifacians & NS & NS & NS & $13.0 \pm 0.8$ & $14.0 \pm 0.6$ & $17.1 \pm 0.7$ & 100 \\
\hline \multirow{7}{*}{ 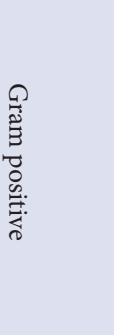 } & S. aureus & NS & NS & $7.3 \pm 0.4$ & $8.1 \pm 0.6$ & $14.2 \pm 0.5$ & $15.2 \pm 0.7$ & 100 \\
\hline & B. subtilis & NS & NS & NS & $8.4 \pm 0.4$ & $10.2 \pm 0.5$ & $17.2 \pm 0.5$ & 250 \\
\hline & B. megaterium & NS & NS & NS & $10.0 \pm 0.8$ & $10.5 \pm 0.4$ & $17.6 \pm 0.5$ & 100 \\
\hline & B. pumilis & NS & $3.2 \pm 0.6$ & $6.5 \pm 0.8$ & $12.4 \pm 0.4$ & $13.5 \pm 0.4$ & $14.2 \pm 0.5$ & 50 \\
\hline & B. cereus & NS & NS & NS & $4.4 \pm 0.4$ & $7.7 \pm 0.7$ & $10.4 \pm 0.4$ & 250 \\
\hline & S. pyogenous & NS & NS & NS & NS & $7.1 \pm 0.6$ & $9.1 \pm 0.6$ & 500 \\
\hline & B. polymyxa & NS & NS & $3.2 \pm 0.8$ & $6.4 \pm 0.6$ & $11.7 \pm 0.7$ & $13.2 \pm 0.5$ & 100 \\
\hline DMSO & Negative control & - & - & - & - & - & - & - \\
\hline
\end{tabular}

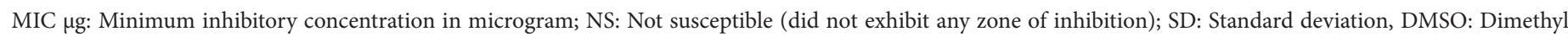
sulfoxide; Values are expressed as mean \pm SD from three experiments $\left({ }^{a} n=3\right)$. 
Al-Samman, et al.: GC-MS/MS Analysis and Antimicrobial Activity of E. officinalis

Table 3: Antibacterial activity of aqueous fruit extract of $E$. officinalis on Gram negative and Gram positive bacteria.

\begin{tabular}{|c|c|c|c|c|c|c|c|c|}
\hline \multirow{2}{*}{\multicolumn{2}{|c|}{ Types and name of organisms }} & \multicolumn{7}{|c|}{ Concentration of extract $(\mu \mathrm{g} / \mathrm{mL})$ and Zone of inhibition $(\mathrm{mm})^{\mathrm{a}} \pm$ SD } \\
\hline & & $25 \mu \mathrm{g}$ & $50 \mu \mathrm{g}$ & $100 \mu \mathrm{g}$ & $250 \mu \mathrm{g}$ & $500 \mu \mathrm{g}$ & $1000 \mu \mathrm{g}$ & MIC $\mu \mathrm{g}$ \\
\hline \multirow{4}{*}{ 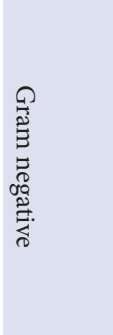 } & E. coli & NS & NS & NS & NS & $5.0 \pm 0.8$ & $19.1 \pm 0.7$ & 500 \\
\hline & V. cholerae & NS & NS & NS & NS & $14.0 \pm 08$ & $15.2 \pm 0.8$ & 500 \\
\hline & S. typhi & NS & $4.3 \pm 0.4$ & $7.2 \pm 0.5$ & $11.2 \pm 0.5$ & $14.0 \pm 0.8$ & $17.6 \pm 0.4$ & 50 \\
\hline & P. alcalifacians & NS & NS & $5.7 \pm 0.5$ & $14.0 \pm 0.7$ & $16.3 \pm 0.2$ & $17.9 \pm 0.3$ & 100 \\
\hline \multirow{4}{*}{ 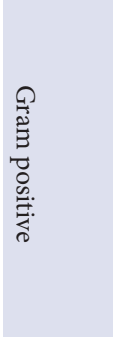 } & S. aureus & NS & $5.5 \pm 0.2$ & $10.8 \pm 0.6$ & $10.9 \pm 0.8$ & $14.7 \pm 0.6$ & $17.8 \pm 0.6$ & 50 \\
\hline & B. subtilis & NS & NS & NS & $13.0 \pm 0.4$ & $14.0 \pm 0.7$ & $18.4 \pm 0.4$ & 250 \\
\hline & B. megaterium & NS & NS & $10.1 \pm 0.6$ & $12.9 \pm 0.7$ & $14.2 \pm 0.5$ & $19.1 \pm 0.6$ & 100 \\
\hline & B. polymyxa & NS & $4.7 \pm 0.3$ & $6.2 \pm 0.5$ & $14.0 \pm 0.6$ & $16.6 \pm 0.2$ & $17.6 \pm 0.3$ & 50 \\
\hline DMSO & Negative control & - & - & - & - & - & - & - \\
\hline
\end{tabular}

MIC $\mu$ : Minimum inhibitory concentration in microgram; NS: Not susceptible (did not exhibit any zone of inhibition); SD: Standard deviation, DMSO: Dimethyl sulfoxide; Values are expressed as mean \pm SD from three experiments $\left({ }^{a} n=3\right)$.

employed in this study for the separation of components was elite PBDME, DTBP and HSTDM [Table 1]. HSTDM was reported first time in EOFE.

\section{Antibacterial and antifungal activity}

EOFE (methanolic and aqueous) have exhibited different degrees of antibacterial activity against seven Gram negative bacteria and seven Gram positive bacteria [Tables 2 and 3]. Similarly, the inhibition zones formed by standard antibiotics (CPF) are shown in [Table 4]. CPF was susceptible at $25 \mu \mathrm{g} / \mathrm{mL}$ against all Gram negative and Gram positive bacteria and exhibit maximum zone of inhibition $(30.2 \pm 0.5 \mathrm{~mm})$ with $V$. cholerae. Maximum resultant zone of inhibition against Gram negative bacteria; P. mirabilis $(8.2 \pm 0.5 \mathrm{~mm})$, S. typhi $(4.3 \pm 0.4 \mathrm{~mm})$ and Gram positive bacteria; $S$. aureus $(5.5 \pm 0.2 \mathrm{~mm}), B$. polymxa $(4.7 \pm 0.3 \mathrm{~mm})$ and B. pumilis $(4.1 \pm 0.3 \mathrm{~mm})$ of aqueous extract were observed at $50 \mu \mathrm{g} / \mathrm{mL}$ where as both Gram negative bacteria; P. aeruginosa, E. coli, V. cholera, S. dysenteriae, P. alcalifaciens and Gram positive bacteria; B. subtilis, $B$. megaterium, B. cereus and $S$. pyogenous were found not susceptible at this concentration. The concentration $(100 \mu \mathrm{g} / \mathrm{mL})$ was found suitable for Gram negative and Gram positive bacteria viz S. typhi $(7.2 \pm 0.5 \mathrm{~mm})$, P. mirabilis $(11.2 \pm 0.5 \mathrm{~mm}), P$. alcalifaciens $(5.7 \pm 0.5 \mathrm{~mm}), S$. aureus $(10.8 \pm 0.62 \mathrm{~m})$, B. megaterium $(10.1 \pm 0.6 \mathrm{~mm})$, B. pumilis $(7.1 \pm 0.4 \mathrm{~mm})$, $B$. cereus $(3.4 \pm 0.4 \mathrm{~mm}), B$. polymyxa $(6.2 \pm 0.3 \mathrm{~mm})$ respectively though no zone of inhibition was observed with Gram negative bacteria; P. aeruginosa, E. coli, V. cholera, S. dysenteriae and Gram positive bacteria; B. subtilis, S. pyogenous respectively. Two Gram negative bacteria (E. coli and $V$. cholerae) were not susceptible while all Gram positive bacteria exhibited considerable margin of zone of inhibition at $250 \mu \mathrm{g} / \mathrm{mL}$ with aqueous extract. Low zone of inhibition was observed with methanolic extract against Gram negative bacteria $P$. mirabilis $(6.2 \pm 0.8 \mathrm{~mm})$ and Gram positive bacteria $B$. pumilis $(3.2 \pm 0.6 \mathrm{~mm})$ while a surprising result was observed with P. aeruginosa, E. coli, V. cholera, S. typhi, S. dysenteriae, $P$. alcalifaciens $S$. aureus, B. subtilis, B. megaterium, B. cereus, S. pyogenous and $B$. polymyxa although it was not able to inhibit the bacterial growth at $50 \mu \mathrm{g} / \mathrm{mL}$. It was confirmed that the concentration $(100 \mu \mathrm{g} / \mathrm{mL})$ was found suitable for Gram negative bacteria $P$. mirabilis $(8.7 \pm 0.6 \mathrm{~mm})$ and Gram positive bacteria $S$. aureus $(7.3 \pm 0.4 \mathrm{~mm}), B$. pumilis $(6.5 \pm 0.8 \mathrm{~mm})$ and $B$. polymyxa $(3.2 \pm 0.8 \mathrm{~mm})$ although it gave negative results for P. aeruginosa, E. coli, V. cholera, S. typhi, S. dysenteriae, P. alcalifaciens, B. subtilis, B. megaterium, B. cereus and S. pyogenous. Clear zone of inhibition was observed against Gram negative; $P$. aeruginosa $(10.1 \pm 0.6 \mathrm{~mm})$, S. typhi $(10.5 \pm 0.8 \mathrm{~mm})$, P. mirabilis $(11.1 \pm 0.8 \mathrm{~mm})$, P. alcalifaciens $(13.0 \pm 0.8 \mathrm{~mm})$ and Gram positive bacteria; S. aureus $(8.1 \pm 0.6 \mathrm{~mm})$, B. subtilis $(8.4 \pm 0.4 \mathrm{~mm}), B$. megaterium $(10.0 \pm 0.8 \mathrm{~mm})$, B. pumilis $(12.4 \pm 0.5 \mathrm{~mm}), B$. cereus $(4.4 \pm 0.4 \mathrm{~mm})$ and $B$. polymyxa $(6.4 \pm 0.6 \mathrm{~mm})$ though no zone of inhibition was ascertained with $E$. coli, V. cholera, S. dysenteriae and S. pyogenous at $250 \mu \mathrm{g} / \mathrm{mL}$. All Gram negative and Gram positive bacteria were found susceptible at very high concentration $(500 \mu \mathrm{g} / \mathrm{mL}-1000 \mu \mathrm{g} / \mathrm{mL})$. The results ascertained two Gram negative bacteria ( $S$. typhi and P. mirabilis) and three Gram positive bacteria ( $S$. aureus, B. pumilis and B. polymyxa) were found susceptible at very low concentration $(50 \mu \mathrm{g} / \mathrm{mL})$ with aqueous extract whereas only one Gram negative bacteria; P. mirabilis and one Gram positive bacteria; B. pumilis was found susceptible $(50 \mu \mathrm{g} / \mathrm{mL})$ with methanolic extract of EOFE [Figure 4].

In Tables 5 and 6 results of antifungal activity of EOFE (aqueous and methanolic) are reported against seven pathogenic fungi to recognize which fruit extracts produced maximum zone of inhibition. Similarly, the inhibition zones formed by standard antifungal (AMT) are shown in [Table 7]. AMT was susceptible at very low concentration $(50 \mu \mathrm{g} / \mathrm{mL})$ against C.albicans and showed maximum zone of inhibition $(5.2 \pm 0.3 \mathrm{~mm})$ and this concentration was found not susceptible against A. niger, N. crassa, P. chrysogenum, T. viridae, A. brasileinsis and C. oxysporum while zone of inhibition was observed at $100 \mu \mathrm{g} / \mathrm{mL}$ against $A$. niger $(2.1 \pm 0.2 \mathrm{~mm}), N$. crassa $(4.1 \pm 0.1 \mathrm{~mm})$, A. brasileinsis $(3.1 \pm 0.2 \mathrm{~mm})$ and C. albicans $(9.3 \pm 0.2 \mathrm{~mm})$. The pathogenic fungi; P. chrysogenum, T. viridae and C. oxysporum not exhibited any zone of inhibition at $100 \mu \mathrm{g} / \mathrm{mL}$. At high concentration $(250 \mu \mathrm{g} / \mathrm{mL})$ AMT exhibited more remarkable zone of inhibition with all pathogenic fungi. Maximum resultant zone of inhibition of aqueous extract $(100 \mu \mathrm{g} / \mathrm{mL})$ were observed against 
Table 4: Antibacterial activity of standard (Ciprofloxacin) on Gram negative and Gram positive bacteria.

\begin{tabular}{|c|c|c|c|c|c|c|c|c|}
\hline \multirow{2}{*}{\multicolumn{2}{|c|}{$\begin{array}{c}\text { Types and name of } \\
\text { organisms }\end{array}$}} & \multicolumn{7}{|c|}{ Concentration of CPF $(\mu \mathrm{g} / \mathrm{mL})$ and Zone of inhibition $(\mathrm{mm})^{\mathrm{a}} \pm$ SD } \\
\hline & & $25 \mu \mathrm{g}$ & $50 \mu \mathrm{g}$ & $100 \mu \mathrm{g}$ & $250 \mu \mathrm{g}$ & $500 \mu \mathrm{g}$ & $1000 \mu \mathrm{g}$ & $\mathrm{MIC} \mu \mathrm{g}$ \\
\hline \multirow{7}{*}{ 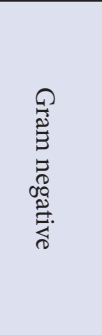 } & E. coli & $26.0 \pm 0.4$ & $29.2 \pm 0.3$ & $32.1 \pm 0.3$ & $34.1 \pm 0.2$ & $38.3 \pm 0.2$ & * & 25 \\
\hline & P. aeruginosa & $30.1 \pm 0.2$ & $32.4 \pm 0.1$ & $34.3 \pm 0.3$ & $35.47 \pm 0.2$ & $38.5 \pm 0.2$ & * & 25 \\
\hline & V. cholerae & $30.2 \pm 0.5$ & $31.1 \pm 0.2$ & $34.4 \pm 0.2$ & $36.2 \pm 0.3$ & $38.0 \pm 0.8$ & * & 25 \\
\hline & S. typhi & $27.2 \pm 0.3$ & $31.4 \pm 0.2$ & $35.4 \pm 0.2$ & $38.3 \pm 0.4$ & $40.3 \pm 0.2$ & * & 25 \\
\hline & S. dysenteriae & $17.3 \pm 0.4$ & $21.4 \pm 0.2$ & $25.1 \pm 0.2$ & $28.2 \pm 0.4$ & $30.3 \pm 0.3$ & * & 25 \\
\hline & P. mirabilis & $16.3 \pm 0.3$ & $19.2 \pm 0.2$ & $22.2 \pm 0.2$ & $26.3 \pm 0.1$ & $31.4 \pm 0.2$ & * & 25 \\
\hline & P. alcalifacians & $20.4 \pm 0.5$ & $22.3 \pm 0.1$ & $26.3 \pm 0.2$ & $29.5 \pm 0.2$ & $32.2 \pm 0.1$ & * & 25 \\
\hline \multirow{7}{*}{ 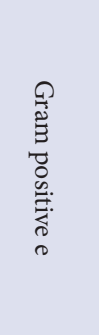 } & S. aureus & $25.1 \pm 0.2$ & $28.1 \pm 0.2$ & $31.4 \pm 0.1$ & $34.3 \pm 0.2$ & $36.3 \pm 0.2$ & * & 25 \\
\hline & B. subtilis & $20.4 \pm 0.5$ & $24.3 \pm 0.2$ & $27.2 \pm 0.3$ & $30.3 \pm 0.1$ & $36.3 \pm 0.2$ & * & 25 \\
\hline & B. megaterium & $19.2 \pm 0.2$ & $26.1 \pm 0.2$ & $28.3 \pm 0.4$ & $30.3 \pm 0.3$ & $36.2 \pm 0.2$ & * & 25 \\
\hline & B. pumilis & $18.2 \pm 0.1$ & $20.1 \pm 0.3$ & $22.1 \pm 0.2$ & $24.3 \pm 0.2$ & $26.3 \pm 0.2$ & * & 25 \\
\hline & B. cereus & $15.4 \pm 0.2$ & $18.1 \pm 0.3$ & $21.1 \pm 0.2$ & $26.2 \pm 0.2$ & $28.2 \pm 0.2$ & * & 25 \\
\hline & S. pyogenous & $19.2 \pm 0.3$ & $24.4 \pm 0.2$ & $26.1 \pm 0.3$ & $29.3 \pm 0.3$ & $34.2 \pm 0.1$ & * & 25 \\
\hline & B. polymyxa & $21.1 \pm 0.2$ & $25.2 \pm 0.1$ & $28.0 \pm 0.7$ & $32.4 \pm 0.1$ & $35.4 \pm 0.2$ & * & 25 \\
\hline DMSO & Negative control & - & - & - & - & - & - & - \\
\hline
\end{tabular}

MIC $\mu \mathrm{g}$ : Minimum inhibitory concentration in microgram; NS: Not susceptible (did not exhibit any zone of inhibition); SD: Standard deviation; DMSO: Dimethyl sulfoxide; CPF: Ciprofloxacin; Values are expressed as mean \pm SD from three experiments $\left({ }^{\mathrm{a}} \mathrm{n}=3\right)$; ${ }^{*}$ Zones could not be measured due to margining.

Table 5: Antifungal activity of methanolic fruit extract of E. officinalis.

\begin{tabular}{|c|c|c|c|c|c|c|c|}
\hline \multirow{2}{*}{$\begin{array}{l}\text { Name of } \\
\text { organisms }\end{array}$} & \multicolumn{7}{|c|}{ Concentration of extract $(\mu \mathrm{g} / \mathrm{mL})$ and Zone of inhibition $(\mathrm{mm})^{\mathrm{a}} \pm S D$} \\
\hline & $25 \mu \mathrm{g}$ & $50 \mu \mathrm{g}$ & $100 \mu \mathrm{g}$ & $250 \mu \mathrm{g}$ & $500 \mu \mathrm{g}$ & $1000 \mu \mathrm{g}$ & MIC $\mu \mathrm{g}$ \\
\hline A. niger & NS & NS & NS & NS & $3.1 \pm 0.3$ & $6.0 \pm 0.4$ & 500 \\
\hline N. crassa & NS & NS & NS & NS & $5.0 \pm 0.4$ & $8.0 \pm 0.4$ & 500 \\
\hline P. chrysogenum & NS & NS & NS & NS & $2.3 \pm 0.2$ & $4.6 \pm 0.2$ & 500 \\
\hline T. viridae & NS & NS & NS & NS & $2.3 \pm 0.3$ & $3.3 \pm 0.2$ & 500 \\
\hline A. brasileinsis & NS & NS & NS & $2.5 \pm 0.1$ & $3.3 \pm 0.2$ & $7.1 \pm 0.3$ & 250 \\
\hline C. albicans & NS & NS & NS & $4.2 \pm 0.1$ & $13.2 \pm 0.2$ & $14.3 \pm 0.2$ & 250 \\
\hline C. oxysporum & NS & NS & NS & NS & NS & $10.4 \pm 0.3$ & 1000 \\
\hline DMSO & - & - & - & - & - & - & - \\
\hline
\end{tabular}

MIC $\mu \mathrm{g}$ : Minimum inhibitory concentration in microgram; NS: Not susceptible (did not exhibit any zone of inhibition); SD: Standard deviation, DMSO: Dimethyl sulfoxide; Values are expressed as mean \pm SD from three experiments $\left({ }^{a} \mathrm{n}=3\right)$.

Table 6: Antifungal activity of aqueous fruit extract of $E$. officinalis.

\begin{tabular}{|c|c|c|c|c|c|c|c|}
\hline \multirow[b]{2}{*}{$\begin{array}{l}\text { Name of } \\
\text { Organism }\end{array}$} & \multicolumn{7}{|c|}{ Concentration of extract $(\mu \mathrm{g} / \mathrm{mL})$ and Zone of inhibition $(\mathrm{mm})^{\mathrm{a}} \pm$ SD } \\
\hline & $25 \mu \mathrm{g}$ & $50 \mu \mathrm{g}$ & $100 \mu \mathrm{g}$ & $250 \mu \mathrm{g}$ & $500 \mu \mathrm{g}$ & $1000 \mu \mathrm{g}$ & MIC $\mu \mathrm{g}$ \\
\hline A. niger & NS & NS & NS & $3.2 \pm 0.1$ & $4.0 \pm 0.1$ & $7.1 \pm 0.3$ & 250 \\
\hline N. crassa & NS & NS & $2.1 \pm 0.1$ & $3.5 \pm 0.1$ & $5.7 \pm 0.6$ & $8.7 \pm 0.5$ & 100 \\
\hline P. chrysogenum & NS & NS & NS & NS & $3.0 \pm 0.3$ & $5.6 \pm 0.2$ & 500 \\
\hline T. viridae & NS & NS & NS & NS & $3.2 \pm 0.1$ & $4.3 \pm 0.2$ & 500 \\
\hline A. brasileinsis & NS & NS & NS & $3.0 \pm 0.2$ & $4.0 \pm 0.3$ & $9.5 \pm 0.5$ & 250 \\
\hline C. albicans & NS & NS & $2.8 \pm 0.2$ & $5.0 \pm 0.2$ & $15.3 \pm 0.3$ & $16.6 \pm 0.7$ & 100 \\
\hline C. oxysporum & NS & NS & NS & NS & $7.2 \pm 0.1$ & $12.4 \pm 0.4$ & 500 \\
\hline DMSO & - & - & - & - & - & - & - \\
\hline
\end{tabular}

MIC $\mu$ g: Minimum inhibitory concentration in microgram; NS: Not susceptible (did not exhibit any zone of inhibition); SD: Standard deviation, DMSO: Dimethyl sulfoxide; Values are expressed as mean \pm SD from three experiments $\left({ }^{a} n=3\right)$. 
Table 7: Antifungal activity of Amphotericin.

\begin{tabular}{|c|c|c|c|c|c|c|c|}
\hline \multirow[t]{2}{*}{ Name of Organism } & \multicolumn{7}{|c|}{ Concentration of AMT $(\mu \mathrm{g} / \mathrm{mL})$ and Zone of inhibition $(\mathrm{mm})^{\mathrm{a}} \pm$ SD } \\
\hline & $25 \mu \mathrm{g}$ & $50 \mu \mathrm{g}$ & $100 \mu \mathrm{g}$ & $250 \mu \mathrm{g}$ & $500 \mu \mathrm{g}$ & $1000 \mu \mathrm{g}$ & MIC $\mu \mathrm{g}$ \\
\hline A. niger & NS & NS & $2.1 \pm 0.2$ & $5.2 \pm 0.2$ & $8.2 \pm 0.2$ & $10.4 \pm 0.1$ & 100 \\
\hline N. crassa & NS & NS & $4.1 \pm 0.1$ & $6.5 \pm 0.1$ & $12.2 \pm 0.3$ & $19.1 \pm 0.4$ & 100 \\
\hline P. chrysogenum & NS & NS & NS & $4.1 \pm 0.2$ & $10.4 \pm 0.1$ & $15.3 \pm 0.2$ & 250 \\
\hline T. viridae & NS & NS & NS & $5.1 \pm 0.2$ & $9.4 \pm 0.1$ & $14.3 \pm 0.2$ & 250 \\
\hline A. brasileinsis & NS & NS & $3.1 \pm 0.2$ & $5.4 \pm 0.3$ & $8.1 \pm 0.2$ & $13.2 \pm 0.3$ & 100 \\
\hline C. albicans & NS & $5.2 \pm 0.3$ & $9.3 \pm 0.2$ & $18.5 \pm 0.1$ & $20.1 \pm 0.3$ & $22.7 \pm 0.7$ & 50 \\
\hline C. oxysporum & NS & NS & NS & $9.3 \pm 0.2$ & $15.2 \pm 0.1$ & $17.4 \pm 0.4$ & 250 \\
\hline DMSO & - & - & - & - & - & - & - \\
\hline
\end{tabular}

MIC $\mu \mathrm{g}:$ Minimum inhibitory concentration in microgram; NS: Not susceptible (did not exhibit any zone of inhibition); SD: Standard deviation; DMSO: Dimethyl sulfoxide; AMT: Amphotericin; Values are expressed as mean \pm SD from three experiments $\left({ }^{a} n=3\right)$.

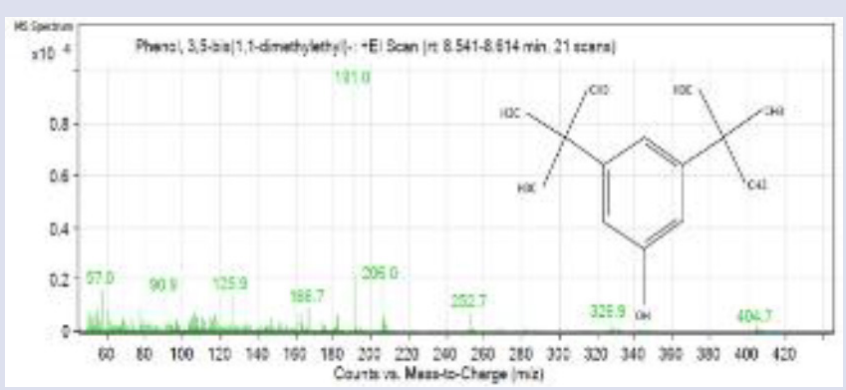

Figure 1: GC-MS/MS Chromatogram, structure and resultant mass peak of methanolic extract of $E$. officinalis showing PBDME Counts vs Mass to Charge $\mathrm{m} / \mathrm{z}(206)[\mathrm{M}+\mathrm{H}]^{+}$; Mobile phase: Helium gas and Nitrogen collision gas; Injection volume (1 mL); Flow rate $(1.0 \mathrm{~mL} / \mathrm{min})$;Oven temperature $\left(70^{\circ} \mathrm{C}\right.$ for $2 \mathrm{~min}$.); Runtime (8.541- $\left.8.614 \mathrm{~min}, 21 \mathrm{scans}\right)$.

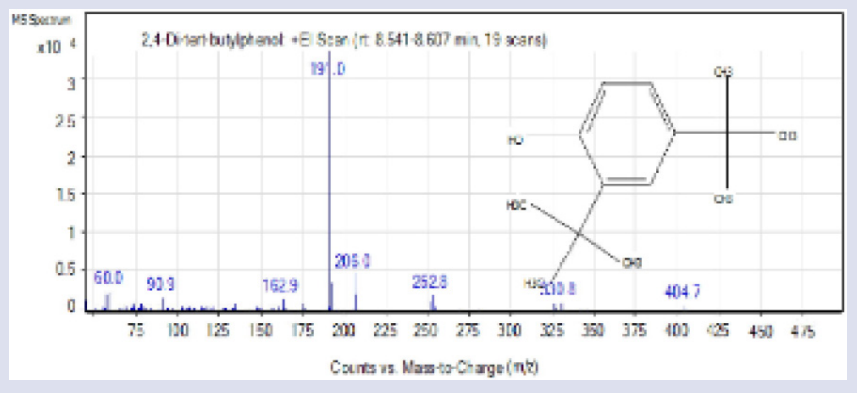

Figure 2: GC-MS/MS Chromatogram, structure and resultant mass peak of aqueous extract of $E$. officinalis showing DTBP Counts vs Mass to Charge $\mathrm{m} / \mathrm{z}(206)[\mathrm{M}+\mathrm{H}]^{+}$; Mobile phase: Helium gas and Nitrogen collision gas; Injection volume ( $1 \mathrm{~mL})$; Flow rate $(1.0 \mathrm{~mL} / \mathrm{min})$; Oven temperature $\left(70^{\circ} \mathrm{C}\right.$ for $2 \mathrm{~min}$.); Run time (8.541- $8.607 \mathrm{~min}, 19$ scans).

C. albicans $(2.8 \pm 0.2 \mathrm{~mm})$ and $N$. crassa $(2.1 \pm 0.1 \mathrm{~mm})$ accept $A$. niger, P. chrysogenum, T. viridae, A. brasileinsis and C. oxysporum while no zone of inhibition of methanolic extract was observed at $100 \mu \mathrm{g} / \mathrm{mL}$ with all pathogenic fungi. The zone of inhibition was observed for A. niger $(3.2 \pm 0.1 \mathrm{~mm}), N$. crassa $(3.5 \pm 0.1 \mathrm{~mm})$, A. brasileinsis $(3.0 \pm 0.2 \mathrm{~mm})$ and C. albicans $(5.1 \pm 0.2 \mathrm{~mm})$ at $250 \mu \mathrm{g} / \mathrm{mL}$ whereas the pathogenic fungi;

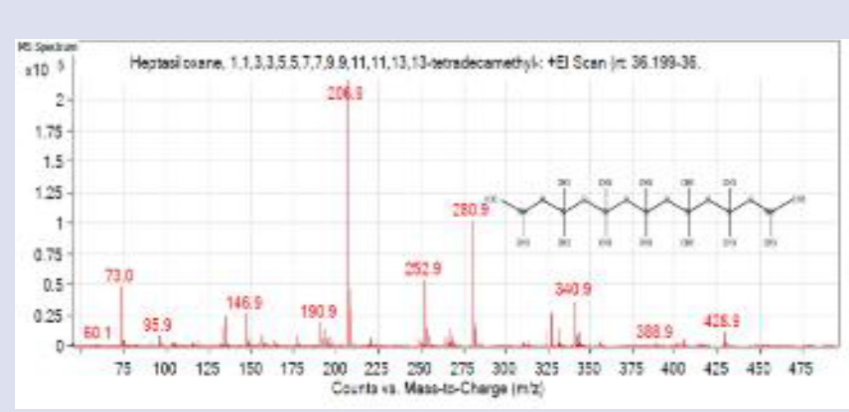

Figure 3: GC-MS/MS Chromatogram, structure and resultant mass peak of aqueous extract of $E$. officinalis showing HSTDM Counts vs Mass to Charge $\mathrm{m} / \mathrm{z}(503)[\mathrm{M}+\mathrm{H}]^{+}$; Mobile phase: Helium gas and Nitrogen collision gas; Injection volume (1 mL); Flow rate $(1.0 \mathrm{~mL} / \mathrm{min})$; Oven temperature $\left(70^{\circ} \mathrm{C}\right.$ for $2 \mathrm{~min}$.); Run time (35.23- $36.199 \mathrm{~min}, 19$ scans).

P. chrysogenum, T. viridae and C. oxysporum were not susceptible at this concentration with aqueous extract. Zone of inhibition of methanolic extract against $C$. albicans $(4.2 \pm 0.1 \mathrm{~mm})$ and A. brasileinsis $(2.5 \pm 0.1 \mathrm{~mm})$ were observed at $250 \mu \mathrm{g} / \mathrm{mL}$ while no zone of inhibition was observed at $250 \mu \mathrm{g} / \mathrm{mL}$ against $A$. niger, N. crassa, P. chrysogenum, T. viridae and C. oxysporum. The fungi A. niger, N. crassa, P. chrysogenum, T. viridae, A. brasileinsis, C. albicans and C. oxysporum were found susceptible with aqueous extract at high concentration $(500 \mu \mathrm{g} / \mathrm{mL})$ whereas only C. oxysporum was not susceptible with methanolic extract at this concentration. In our study, it was marked that aqueous extract of EOFE has good antifungal activity at low concentration $(100 \mu \mathrm{g} / \mathrm{mL})$ while methanolic extract exhibited minimal antifungal activity at $(250 \mu \mathrm{g} / \mathrm{mL})$ [Figure 5]. It was amazing that aqueous extract showed a potent inhibitory effect for Gram negative bacteria such as (P. mirabilis) with MIC value of $(50 \mu \mathrm{g} / \mathrm{mL})$ and zone of inhibition $(8.2 \pm 0.5 \mathrm{~mm})$; two Gram positive bacteria (B. pumilis and B. polymyxa) with MIC value of MIC of $(50 \mu \mathrm{g} / \mathrm{mL})$ and zone of inhibition $(4.1 \pm 0.3 \mathrm{~mm})$ and $(4.7 \pm 0.3 \mathrm{~mm})$ respectively in comparison to methanolic extract at the same MIC. Methanolic extract confirmed low antifungal activity, However broad antifungal effect of aqueous extract was observed with $N$. crassa. All these results confirmed aqueous extract as broad spectrum antimicrobial agent and inhibit the growth of wide range of Gram negative, Gram positive bacteria and fungi. 


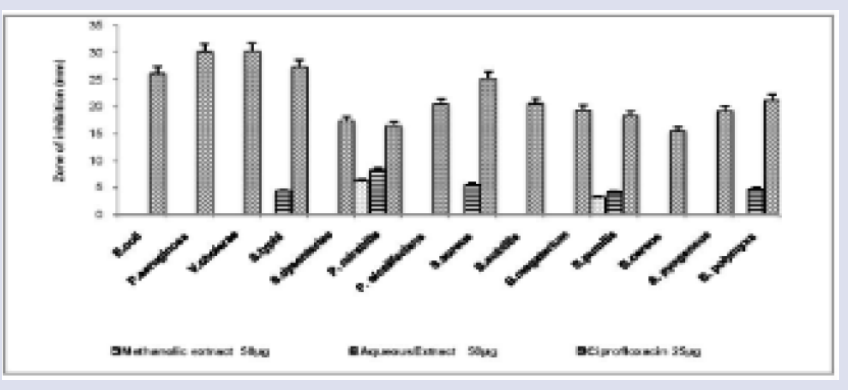

Figure 4: Standard (Ciprofloxacin) and E. officinalis extracts (methanolic and aqueous) graph which displayed the most comprehensive minimum inhibitory concentration (MIC) and number of bacteria against which they were active at different doses. Standard is more susceptible at $25 \mu \mathrm{g}$ concentration against all Gram negative and Gram positive bacteria and give maximum zone of inhibition $(30.2 \pm 0.5 \mathrm{~mm}$ with $V$. cholerae). Maximum resultant zone of inhibition $(8.2 \pm 0.5 \mathrm{~mm}, 4.3 \pm 0.4 \mathrm{~mm}, 5.5 \pm 0.2 \mathrm{~mm}$, $4.7 \pm 0.3 \mathrm{~mm}$ and $4.1 \pm 0.3 \mathrm{~mm}$ ) of aqueous extract is observed at $50 \mu \mathrm{g}$ concentration against Gram negative (P. mirabilis, S. typhi) and Gram positive (S. aureus, B. polymxa and B. pumilis) bacteria. Low margin of zone of inhibition of methanolic extract is observed $(6.2 \pm 0.8 \mathrm{~mm}$ and $3.2 \pm 0.6 \mathrm{~mm})$ at $50 \mu \mathrm{g}$ concentration with Gram negative (P. mirabilis) and Gram positive (B. pumilis), while no margin of zone of inhibition of methanolic extract is observed with S. typhi, S. aureus and B. polymxa.

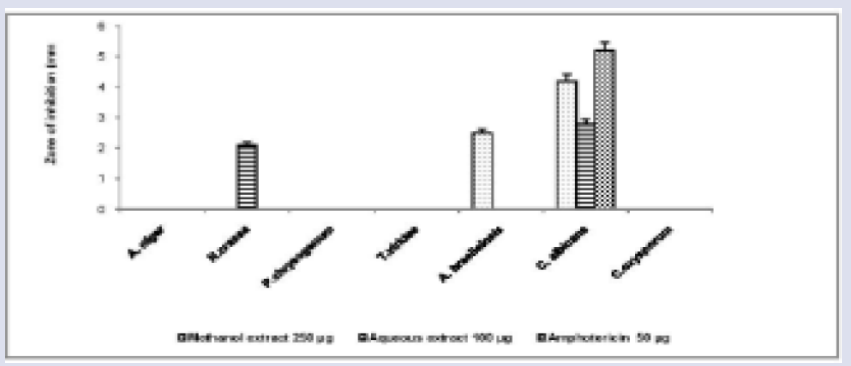

Figure 5: Standard (Amphotericin) and E. officinalis extracts (Methanolic/Aqueous) graph which displayed the most comprehensive minimum inhibitory concentration (MIC) and the number of fungi against which they were active at different concentration. Standard is more susceptible at $50 \mu \mathrm{g}$ concentration against $C$. albicans and give maximum zone of inhibition $(5.2 \pm 0.3 \mathrm{~mm})$. Maximum resultant zone of inhibition $(2.8 \pm 0.2 \mathrm{~mm}$ and $2.1 \pm 0.1 \mathrm{~mm}$ ) of aqueous extract is observed at $50 \mu \mathrm{g}$ concentration against $C$. albicans and $N$. crassa fungi while no margin of zone of inhibition of methanolic extract is observed at $50 \mu \mathrm{g}$ concentration with all fungi tested. Zone of inhibition of methanolic extract is observed $(4.2 \pm 0.1 \mathrm{~mm}$ and $2.5 \pm 0.1 \mathrm{~mm})$ at $250 \mu \mathrm{g}$ concentration against $C$. albicans and $A$. brasileinsis.

\section{DISCUSSION}

UAE is a novel technique used to extract bioactive compounds from medicinal plants. The same technique was employed in this study for the extraction of bioactive compounds with different solvent (methanol and water) from the fruit of E. officinalis. This is an efficient method, that requires very short time, less solvent, reduced solvent exposure, eco-friendly and is comparatively simple to operate. It minimizes the risk of oxidation, disintegration and decomposition of chemical constituents of plant. ${ }^{6}$ In this method, cavitations and bubbles are produced that exerts strong shear significant to fragment and disrupt plant tissues and cell walls leading to enhanced mass transfer of the cell contents. During the UAE process, extraction efficiency is improved due to increase in surface areas, rate of mass transfer and production of cavitations and bubbles. ${ }^{12-15}$ optimization and demonstration of an ultrasonic assisted extraction (UAE Because of these advantages, the UAE techniques have become one of the best techniques for the extraction of bioactive constituents from medicinal plants. In this study, UAE was efficiently used for the preparation of aqueous and methanolic extracts of E. officinalis fruits. The extraction was carried out at $20^{\circ} \mathrm{C}$, for $20 \mathrm{~min}$ using a solid-to-solvent ratio of 1: $60 \mathrm{w} / \mathrm{v}$. The percentage yield was found to be $41.33 \% \mathrm{w} / \mathrm{v}$ and $23.0 \% \mathrm{w} / \mathrm{v}$ with water and methanol, respectively. On the basis of these results, the study concludes that UAE is suitable for the extraction of phytoconstituents from medicinal plants. GC-MS/MS is a frequently used analytical technique for the quantification of phytoconstituents including polyphenols from plant extracts due to its accuracy, reliability and repeatability. In an earlier study, DTBP was detected by GC-MS analysis and was found to be a potential antifungal agent. Another report indicated that $100 \mu \mathrm{g} / \mathrm{mL}$ of DTBP prevent spore germination of $F$. oxysporum and was detected by GC-MS analysis. Moreover, PBDME, DTBP and HSTDM were also reported to possess antibacterial, antifungal, antioxidant and anticancer potential. ${ }^{16-17}$ Recently, PBDME, DTBP and HSTDM have been reported to decrease the growth of $S$. marcescens, a Gram negative bacteria. ${ }^{18}$ Furthermore, the antifungal activities exhibited by DTBP was investigated by Varsha et al. ${ }^{17}$ their finding was in total harmony with the previous report, in which the phenolic nature of PBDME, DTBP was found to alter the cell membrane integrity of bacteria. ${ }^{19}$ The result clearly showed that the antimicrobial activity of EOFE was due to presence of PBDME, DTBP and HSTDM and confirmed these compounds as an ideal antimicrobial agent. This comprehensive study supports and confirms previous results on antifungal potential of EOFE. ${ }^{20}$ The results of the study demonstrated that volatiles present in EOFE inhibited the congregation of spindle microtubules and modified the chromosomal arrangement at the metaphase leading to chromatid loss, which led to reduce the germination of spores. The findings confirmed the previous reports as well as explained the efficiency of these compounds in prevention of fungal growth. Still, to the best of our knowledge; these compounds have hardly ever been documented for antibacterial and antifungal activity against such collection of microbes. The mode of action of phenoliocs compounds is not fully clear and noticeably speculated to involve membrane distraction by means of active compound. ${ }^{21}$ Antibacterial and antifungal activity of the EOFE was quantitatively assessed on the basis of zone of inhibition. In the current study, the inhibitory potential of EOFE was evaluated against seven Gram negative bacteria; P. aeruginosa, E. coli, V. cholera, S. typhi, S. dysenteriae, P. mirabilis, P. alcalifaciens and seven Gram positive bacteria; S. aureus, B. subtilis, B. megaterium, B. pumilis, B. cereus, $S$. pyogenous and $B$. polymyxa. A remarkable antibacterial activity with $\mathrm{CPF}$ and EOFE was observed against pathogenic bacteria for which they were active at different concentration taking DMSO as negative control. All the Gram positive bacteria were susceptible to CPF at $25 \mu \mathrm{g} / \mathrm{mL}$ and exhibited maximum zone of inhibition with $V$. cholerae. Both Gram negative and Gram positive bacteria were found susceptible to EOFE at concentration $(500 \mu \mathrm{g} / \mathrm{mL}-1000 \mu \mathrm{g} / \mathrm{mL})$. The results ascertained two Gram negative bacteria ( $S$. typhi and $P$. mirabilis) and three Gram positive bacteria ( $S$. aureus, B. pumilis and B. polymyxa) were found susceptible at very low concentration $(50 \mu \mathrm{g} / \mathrm{mL})$ to aqueous extract whereas only one Gram negative bacteria; P. mirabilis and one Gram positive bacteria; $B$. pumilis was found susceptible $(50 \mu \mathrm{g} / \mathrm{mL})$ to methanolic extract of EOFE. For antifungal activity the inhibitory potential of EOFE was evaluated against seven pathogenic fungi strains; A. niger, N. crassa, 
P. chrysogenum, T. viridae, A. brasileinsis, C. albicans and C. oxysporum. Here, the potency of EOFE was compared with AMT. Among the tested fungal strains, only $N$. crassa and C. albicans showed moderate inhibition with aqueous extract at $100 \mu \mathrm{g} / \mathrm{mL}$ while no inhibition was observed with methanolic extract at $100 \mu \mathrm{g} / \mathrm{mL}$. C. albicans and A. brasileinsis were found to be susceptible to methanolic extract at $250 \mu \mathrm{g} / \mathrm{mL}$. The fungi A. niger, N. crassa, P. chrysogenum, T. viridae, A. brasileinsis, C. albicans and C. oxysporum were found susceptible to aqueous extract at high concentration $(500 \mu \mathrm{g} / \mathrm{mL})$ whereas only C. oxysporum was found to be not susceptible with methanolic extract at this concentration. The results of the current study revealed that there was marked antibacterial and anti-fungal inhibition with the aqueous extract of EOFE at low concentration $(100 \mu \mathrm{g} / \mathrm{mL})$ while methanolic extract exhibited minimal antifungal activity at $250 \mu \mathrm{g} / \mathrm{mL}$. The aqueous extract also inhibited the growth of $N$. crassa. The existence of antifungal potential only in crude extracts may be due to the synergistic power of various bioactive constituents in the both extracts. The antimicrobial activities of EOFE can be explained on the basis of its GC-MS/MS analysis. In this study, the results revealed the broad spectrum of antibacterial and antifungal activity in crude extract of EOF may be due to the synergistic influence of various bioactive compounds in EOFE. In a nutshell, EOFE showed their potential antimicrobial activities against the bacterial and fungal pathogens and provides an ample opportunity to plant based drug designing based on ethnomedicine. The possibility of using EOFE in folk medicine for the management of infectious diseases is validated by this study.

\section{CONCLUSION}

In summary, the results of this study showed that both the methanolic and aqueous extracts exhibit antibacterial and antifungal properties due to their bioactive compounds. To our knowledge, this is the first report of its activity against such collection of Gram negative bacteria; $P$. alcalifaciens and Gram positive bacteria; B. pumilis, B. polymyxa and fungal strains; N. crassa, A. brasileinsis and C. oxysporum. Furthermore, use of $\mathrm{UAE}$ to extract vital bioactive extract as EOFE worth mentioning. The considerable inhibitory effect of EOFE against various microbial species was mainly due to the presence of phenolic compounds and other phytocompounds including PBDME, DTBP and HSTDM compound. With this milieu, the present study was focussed towards analysing the value of EOFE against various pathogens microbes. The results revealed excellent antimicrobial activity of aqueous extract and methanolic extracts of E. officinalis.

\section{ACKNOWLEDGEMENT}

The authors gratefully acknowledge the Bioactive Natural Compound Laboratory (BNCL), Department of Pharmacognosy and Phytochemistry, Glocal School of Pharmacy, Glocal University, Saharanpur 247121, U.P, India providing the experimental facilities. The authors would like to thanks Ms. Kahkashan for their support and valuable suggestions to improve the quality of the manuscript.

\section{FUNDING}

This research received no specific grant from any funding agency in the public, commercial or not-for-profit sectors.

\section{CONFLICT OF INTEREST}

The authors declare no conflict interest.

\section{ABBREVIATIONS}

BNCL: Bioactive Natural Compound Laboratory; UAE: Ultrasonic assisted extraction; EOFE: Emblica officinalis fruit extract; RT: Retention Time; Mol.Wt.: Molecular weight; MIC $\mu$ g: Minimum inhibitory concentration in microgram; NS: Not susceptible (did not exhibit any zone of inhibition); SD: Standard deviation; DMSO: Dimethyl sulfoxide; CPF: Ciprofloxacin; AMT: Amphotericin; DTBP: 2,4-Di-tert-butylphenol; PBDME: Phenol,3,5-bis1,1dimethylethyl; HSTDM: Heptasilox ane1,1,3,3,5,5,7,7,9,9,11,11,13,13-tetradecamethyl.

\section{REFERENCES}

1. Khan KH. Role of Emblica officinalis in Medicine - A Review. Rev Bot Res Int 2009;4(2):218-28.

2. Kim HJ, Yokozawa T, Kim HY, Tohda C, Rao TP, Juneja LR. Influence of Amla (Emblica officinalis Gaertn.) on Hypercholesterolemia and Lipid Peroxidation in Cholesterol-Fed Rats. J Nutr Sci Vitaminol (Tokyo). 2005;51(6):413-8.

3. Pasanen A, Pasanen P. Volatile Organic Metabolites Associated With Some Toxic Fungi and Their Mycotoxins. Analyst. 1996;121(12):1949-53.

4. Saeed S, Tariq P. Antimicrobial activities of Emblica officinalis and Coriandrum sativum against gram positive bacteria and Candida albicans. Pakistan J Bot. 2007;39(3):913-7.

5. Darshan Dharajiya, Tarun Khatrani, Payal Pate NM. Evaluation of antifungal activity of Emblica officinalis, Aloe vera and Vitex negundo extracts. J Chem Biol Phys Sci. 2015;5(4):3990-6.

6. Puri M, Sharma D, Barrow CJ. Enzyme-assisted extraction of bioactives from plants. Trends Biotechnol. 2012;30(1):37-44.

7. Banjoo DR, Nelson PK. Improved ultrasonic extraction procedure for the determination of polycyclic aromatic hydrocarbons in sediments. J Chromatogr A 2005;1066(1-2):9-18

8. Zhang HF, Yang XH, Zhao LD, Wang Y. Ultrasonic-assisted extraction of epimedin $\mathrm{C}$ from fresh leaves of Epimedium and extraction mechanism. Innov Food Sci Emerg Technol. 2009;10(1):54-60.

9. Balasubramanian S, Ganesh D, Panchal P, Teimouri M, Surya VVS GC-MS analysis of phytocomponents in the methanolic extract of Emblica officinalis Gaertn (Indian Gooseberry). J Chem Pharm Res. 2014;6(6):843-5.

10. Ergin MA, Cemil I, Talip C, Hami A. High hydrostatic pressure extraction of phenolic compounds from Maclura pomifera fruits. African J Biotechnol. 2012;11(4):930-7.

11. Santana CM, Ferrera ZS, Padrón MET, Rodríguez JJS. Methodologies for the extraction of phenolic compounds from environmental samples: New approaches. Molecules. 2009;14(1):298-320.

12. Bossio JP, Harry J, Kinney CA. Application of ultrasonic assisted extraction of chemically diverse organic compounds from soils and sediments. Chemosphere. 2008;70(5):858-64.

13. Rostagno MA, Palma M, Barroso CG. Ultrasound-assisted extraction of soy isoflavones. J Chromatogr A. 2003;1012(2):119-28.

14. Toma M, Vinatoru M, Paniwnyk L, Mason TJ. Investigation of the effects of ultrasound on vegetal tissues during solvent extraction. Ultrason Sonochem. $2001 ; 8(2): 137-42$.

15. Wang LC. Ultrasonic Peptization of Soybean Proteins From Autoclaved Flakes, Alcohol-Washed Flakes and Commercial Samples. J Food Sci. 1978;43(4):1311-3.

16. Choi SJ, Kim JK, Kim HK, Harris K, Kim CJ, Park GG, et al. 2,4-Di- tert -butylphenol from Sweet Potato Protects Against Oxidative Stress in PC12 Cells and in Mice. J Med Food. 2013;16(11):977-83.

17. Varsha KK, Devendra L, Shilpa G, Priya S, Pandey A, Nampoothiri KM. 2,4-Ditert-butyl phenol as the antifungal, antioxidant bioactive purified from a newly isolated Lactococcus sp. Int J Food Microbiol. 2015;211:44-50.

18. Padmavathi AR, Abinaya B, Pandian SK. Phenol, 2,4-bis(1,1-dimethylethyl) of marine bacterial origin inhibits quorum sensing mediated biofilm formation in the uropathogen Serratia marcescens. Biofouling. 2014;30(9):1111-22.

19. Rangel-Sánchez G, Castro-Mercado E, García-Pineda E. Avocado roots treated with salicylic acid produce phenol-2,4-bis (1,1-dimethylethyl), a compound with antifungal activity. 2014;171(3-4):189-98.

20. Yuan J, Raza W, Shen Q, Huang Q. Antifungal activity of bacillus amyloliquefaciens NJN-6 volatile compounds against Fusarium oxysporum f. sp. cubense. Appl Environ Microbiol. 2012;78(16):5942-4

21. Cowan M. Plant Products as Antimicrobial Agents. Cosmet Sci Technol. 2006;12(4):564-82. 
GRAPHICAL ABSTRACT
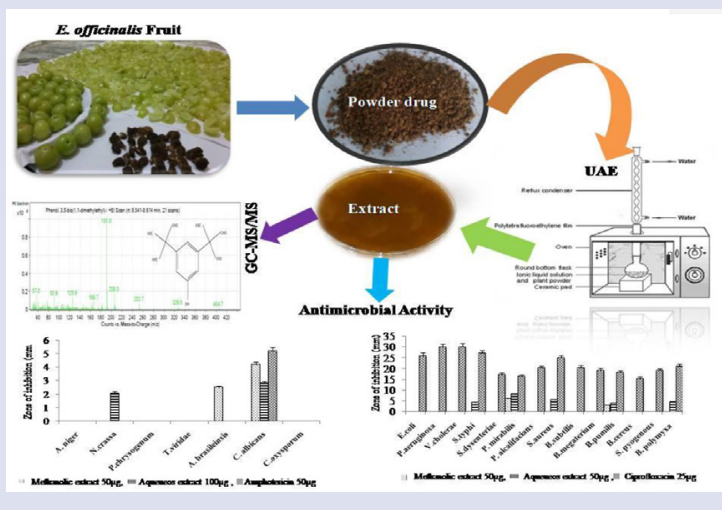

\section{SUMMARY}

- Emblica officinalis is a well-known folk medicinal plant used Indian traditional medicines for over years. Its use in the ethno medicines is well acknowledged. It belongs to the Euphorbiaceae family. UAE technique is used for the extraction of plant material. GC-MS/MS analysis confirmed the presence of PBDME DTBP and HSTDM. For best of our knowledge in E. officinalis fruit extract, the antimicrobial activity of PBDME, DTBP and HSTDM has not been reported earlier in aqueous and methanolic extract. Potential antimicrobial activity was confirmed in aqueous extract which might be due to the presence of phenolics compounds present in it. The present study contributed in analysing the phytoconstituents by GC-MS/MS analysis which are responsible for various antibacterial and antifungal activities.

Cite this article: Al-Samman AMMA, Kahkashan, Siddique NA. Gas Chromatography-Mass Spectrometry (GC-MS/MS) Analysis, UItrasonic Assisted Extraction, Antibacterial and Antifungal Activity of Emblica officinalis Fruit Extract. Pharmacog J. 2019;11(2):315-23. 